\title{
VASCULAR ACCESS
}

Vascular access involves inserting a catheter into an appropriate blood vessel in order to measure useful diagnostic parameters, draw blood for diagnostic testing, and/or provide specific therapeutic interventions.

Vascular access procedures were performed in approximately 417,000 discharges in 2002, according to the Healthcare Cost and Utilization Project (HCUP). Many hospitalized patients require vascular access, and hospitalists will differentiate patients who simply need peripheral venous access from those who require more invasive types of arterial or central venous access. Complications of vascular catheters can cause prolonged hospital stays and increase morbidity and mortality. Hospitalists advocate for patients to determine the most appropriate type of vascular access based on the patient's diagnostic and therapeutic requirements and overall clinical condition.

\section{KNOWLEDGE}

Hospitalists should be able to:

- Name the various locations for peripheral venous access and describe the normal vasculature and surrounding anatomy of the site chosen for access.

- Name the various locations for arterial or central venous access and describe the normal vasculature and surrounding anatomy of the site chosen for vascular access.

- Describe the collateral flow for arterial access procedures.

- Describe the clinical findings or disease processes that require arterial or central venous access.

- Explain the role of ultrasonography in vascular access placement.

- Explain indications and contraindications of the various arterial or central venous access procedures.

- Describe and differentiate the potential risks and complications of individual vascular access procedures based on the site chosen and other risk factors.

- Select the necessary equipment to perform the indicated vascular access procedure at the bedside.

\section{SKILLS}

Hospitalists should be able to:

- Elicit an accurate and thorough history to identify co-morbid conditions and risk factors for complications related to arterial or central venous vascular access placement.

- Identify absolute and relative contraindications to placement of arterial access or central venous access at specific sites.

- Perform a directed physical examination of the site(s) intended for vascular access.

- Perform specific maneuvers to evaluate for collateral flow for arterial access procedures.

- Properly position the patient and identify anatomic landmarks to obtain vascular access.

- Use sterile techniques during preparation for and performance of vascular access procedures.

- Anticipate and manage complications from the vascular access procedure and in-dwelling catheter.

- Identify and manage the complications of vascular access procedures, which may include infection, thrombotic, and mechanical complications.

- Order and interpret platelet and coagulation studies when indicated.

\section{ATTITUDES}

Hospitalists should be able to:

- Communicate with patients and families to explain the indications and alternatives to vascular access.

- Communicate with patients and families to explain the procedure, its expected therapeutic benefits and potential complications; and to obtain informed consent.

- Provide education to patients and their families regarding the care of long-term vascular access.

- Recognize the importance of proper positioning during the procedure.

- Remove all central venous catheters and arterial catheters as soon as they are no longer needed.

- Promote the use of peripheral venous access over central venous access whenever possible.

- Manage patient discomfort or pain during and after the procedure. 
- Recognize the indications for specialty consultation, which may include interventional radiology, surgery, or critical care medicine.

- Arrange appropriate care for patients being discharged with long-term vascular access.

\section{SYSTEM ORGANIZATION AND IMPROVEMENT}

To improve efficiency and quality within their organizations, Hospitalists should:

- Lead, coordinate or participate in multidisciplinary initiatives to promote patient safety and optimize resource utilization.

- Lead, coordinate or participate in development of IV access teams to improve the placement and maintenance of IV catheters.

- Lead, coordinator or participate in quality improvement programs to monitor hospitalists' performance and/or supervision of vascular access.

- Lead, coordinate or participate in implementation of standard nursing protocols for catheter care.

- Lead, coordinate or participate in efforts to organize and consolidate equipment in an identifiable location in the hospital that is easily accessible to clinicians who perform the procedure. 